\title{
Adenine and guanine nucleotide-specific succinyl-CoA synthetases in the clonal beta-cell mitochondria: implications in the beta-cell high-energy phosphate metabolism in relation to physiological insulin secretion
}

\author{
A. Kowluru \\ Department of Pharmaceutical Sciences, College of Pharmacy and Allied Health Professions, \\ Wayne State University and Beta-cell Biochemistry Research Laboratory, John D. Dingell VA Medical Center, Detroit
}

\section{Abstract}

Aims/hypothesis. Succinyl-CoA synthetase catalyses the substrate level phosphorylation of ADP or GDP. It also supplies succinyl-CoA for heme synthesis. Recently, two distinct mitochondrial succinyl-CoA synthetase activities, one specific for ATP and the other for GTP, have been characterized in various tissues of pigeon. Because of the relative importance of mitochondrial high-energy phosphate metabolism in physiological insulin secretion and the few data available on mitochondrial succinyl-CoA synthetase in the beta cell, this study examined whether ATP-specific and GTP-specific succinyl-CoA synthetase activities are localized in the clonal beta-cell mitochondria.

Methods. Using the mitochondrial extracts from clonal beta [INS-1 and HIT-T15] cells, we measured the formation of succinyl-CoA from succinate, CoA and ATP or GTP. To confirm the identity of these two enzymes, individual subunits of ATP-specific and GTPspecific to succinyl-CoA synthetase were identified by Western blot analysis.

Results. Both ATP-and GTP activities of succinylCoA synthetase were observed in the mitochondrial fractions from these cells. The ratios of GTP to ATP activities of succinyl-CoA synthetase were near unity in both of the cell types studied. Using affinity-purified antisera directed specifically against individual ( $\alpha$ and $\beta$ ) subunits of succinyl-CoA synthetase, we also identified both ATP-specific and GTP-specific forms of succinyl-CoA synthetase in HIT and INS cell mitochondria. Furthermore, using $\left[\gamma^{32} \mathrm{P}\right] \mathrm{ATP}$ as a phosphoryl donor, we observed that the alpha subunit of succinyl-CoA synthetase undergoes autophosphorylation at a histidine residue; co-provision of exogenous succinate and $\mathrm{CoA}$ resulted in pronounced dephosphorylation of the phosphorylated alpha subunit of succinyl-CoA synthetase.

Conclusion/interpretation. We provide evidence for the localization of two distinct activities of succinylCoA synthetase in the beta cell mitochondria. Whereas it is well established that ATP is critical for the beta cell mitochondrial metabolism, we propose that GTP generated by the activation of succinylCoA synthetase could promote key functional roles in the mitochondrial metabolism leading to insulin secretion. [Diabetologia (2001) 44: 89-94]

Keywords Beta cell, succinyl-CoA synthetase, nucleoside diphosphate kinase, mitochondria, ATP, GTP.
Received: 17 July 2000 and in revised form: 24 August 2000

Corresponding author: Dr. A. Kowluru, Department of Pharmaceutical Sciences, College of Pharmacy and Allied Health Professions, 619 Shapero Hall, Wayne State University, Detroit, MI 48202, USA

Abbreviations: SCS, Succinyl-CoA synthetase; A-SCS, ATPspecific succinyl-CoA synthetase; G-SCS, GTP-specific succinyl-CoA synthetase; G-proteins, guanosine triphosphate binding proteins; NDPK, nucleoside diphosphate kinase.
Succinyl-CoA synthetase (SCS) catalyses the substrate level phosphorylation of ADP or GDP. The hydrolysis of the thioester bond of succinyl-CoA is coupled to the phosphorylation of GDP (or ADP) as indicated below:

Succinyl CoA $+\mathrm{P}_{\mathrm{i}}+\mathrm{GDP}[$ or $\mathrm{ADP}] \leftrightarrows$ Succinate + GTP [or ATP] + CoA

This is the only known step in the citric acid cycle that directly yields a high-energy phosphate bond $[1,2]$. Alternatively, SCS catalyses the reverse reac- 
tion to supply succinyl-CoA for heme synthesis and ketone body activation [1,2]. Typically, the SCS is comprised of alpha beta heterodimeric subunits and the alpha subunit undergoes phosphorylation at a histidine residue as a part of the catalytic cycle [3]. Recently, both GTP-specific (EC 6.2.1.4) and ATP-specific (EC. 6.2.1.5) forms of SCS have been reported in various tissues of pigeon $[4,5]$. These are two distinct gene products and the sequence analyses showed that both isoforms of SCS share the same alpha subunit, which undergoes phosphorylation at a histidine residue $[4,5]$ but dissimilar beta subunits. It was, therefore, postulated that the beta subunit determines the nucleotide specificity of the enzyme whereas the alpha subunit contributes to the binding site [4,5]. Based on quantitative measurements of SCS activity in the direction of succinyl-CoA formation, these investigators also reported differential tissue distribution of these activities in pigeon. The ratios of GTP-SCS to ATP-SCS were found to be 7,1, and 0.1 for the kidney, brain, and heart, respectively $[4,5]$.

Very little is known about the nature of SCS in the pancreatic beta cell. A previous report [6] referred to the GTP-specific SCS activity in the context of a possible mechanism underlying insulinotropic effects of monomethyl succinate. These investigators speculated that the insulinotropic effects of monomethyl succinate could result from an increase in the mitochondrial succinyl-CoA and a subsequent decrease in the concentration of GTP activated by mitochondrial SCS [6].

Owing to the lack of data on the islet SCS and to our interest in understanding the roles of protein histidine phosphorylation in beta cell stimulus-secretion coupling [7-9], we quantified SCS activity in the mitochondrial fraction isolated from clonal beta cells and we aimed to determine whether or not ATP or GTPspecific SCS activities are present in these fractions. We give evidence indicating that both A-SCS and GSCS activities are localized in the mitochondrial fraction. We supported these data by immunologic detection of their common alpha, but distinct beta subunits of these enzymes in the cognate fractions.

\section{Materials and methods}

We purchased $\left[\gamma_{-}{ }^{32} \mathrm{P}\right]$ ATP $(22.2 \mathrm{TBq})$ from NEN-Dupont (Boston, Mass., USA). Succinate, CoA, unlabelled ATP and GTP were obtained from Sigma (St. Louis, Mo., USA). Electrophoresis and protein determination reagents were purchased from Bio-Rad (Herecules, Calif., USA). The chemiluminescence (ECL) kit was purchased from Amersham (Chicago, Ill., USA). All other reagents were of the highest purity available.

Antisera directed against ATP- and GTP-specific SCS. An antiserum directed against porcine heart SCS holoenzyme was a generous gift from Dr. W. A. Bridger and Dr. W.T. Wolodko (University of Alberta, Edmonton, Canada). This antiserum recognizes both the alpha and beta subunits of SCS (see text). We also used specific antisera (kindly provided by Dr. Lambeth) directed against the alpha subunit (common to both forms) and the beta subunits directed against ATP-specific and GTP-specific SCS $[4,5]$.

Isolation of mitochondrial fractions from HIT-T15 and INS-1 cells. The HIT-T15 cells were provided by Dr. P. Robertson (Pacific Northwest Research Institute, Seattle, Washington, United States) and INS-1 cells were provided by Dr. C. Wollheim (University of Geneva, Geneva, Switzerland). The mitochondrial fraction from HIT and INS cells was isolated by a differential centrifugation procedure as described previously by us using a Beckman Optima TL-100 ultracentrifuge [8,10-12]. Procedures were carried out at $4{ }^{\circ} \mathrm{C}$ unless stated otherwise. Briefly, homogenates (corresponding to $10-15 \mathrm{mg}$ protein) were prepared in $230 \mathrm{mmol} / \mathrm{l}$ mannitol, $70 \mathrm{mmol} / \mathrm{su}-$ crose/ $5 \mathrm{mmol} / \mathrm{l}$ HEPES buffer, $\mathrm{pH} 7.4$ containing $1 \mathrm{mmol} / \mathrm{l}$ EGTA, $1 \mathrm{mmol} / 1$ dithiothreitol (DTT), $2.5 \mu \mathrm{g} / \mathrm{ml}$ of leupeptin and pepstatin. The homogenates were spun at $600 \mathrm{~g}$ for $5 \mathrm{~min}$ to remove the nuclear and cell-debris pellet. The resulting supernatant was centrifuged at $5500 \mathrm{~g}$ for $10 \mathrm{~min}$ to yield a pellet enriched in mitochondria, which was washed twice with the homogenization medium and resuspended in a suitable volume of the buffer for biochemical studies. Purity of this fraction was judged by quantifying the enrichment of two mitochondrial marker enzymes, namely the succinic-INT-reductase and the cytochrome $c$ oxidase. Additional methodological details of marker-enzyme assays are described in our earlier publications [7-12].

Studies of mitochondrial ATP-and GTP-specific succinyl-CoA synthetases: phosphorylation of SCS. The phosphorylation reaction was carried out in a total volume of $100 \mu \mathrm{l}$ in a buffer consisting of $50 \mathrm{mmol} / \mathrm{l}$ TRIS-HCl, $\mathrm{pH} 7.4,2 \mathrm{mmol} / \mathrm{l}$ DTT, mitochondrial protein and $\left[\gamma_{-}{ }^{32} \mathrm{P}\right] \mathrm{ATP}$ at $37^{\circ} \mathrm{C}$ for different time periods as indicated in the text. The reaction was terminated by adding Laemmli stop solution. Labelled proteins were separated by SDS-PAGE and identified by autoradiography $[7,8]$. Molecular weights of labelled proteins were determined based on relative mobility rates using authentic molecular weight standards from Bio-Rad.

Catalytic activity of SCS. The SCS activity was assayed in the direction of succinyl -CoA formation by a spectrophotometric assay of [13]. The reaction mixture $(500 \mu \mathrm{l})$ contained: $50 \mathrm{mmol} / \mathrm{l}$ succinate, $10 \mathrm{mmol} / 1 \mathrm{MgCl}_{2}, 100 \mu \mathrm{mol} / \mathrm{l}$ ATP or GTP, $100 \mu \mathrm{mol} / \mathrm{l} \mathrm{CoA}$ and $110 \mathrm{mmol} / \mathrm{l} \mathrm{TRIS}-\mathrm{HCl}, \mathrm{pH}$ 7.4. The contents of the reaction mixture were pre-incubated at $30^{\circ} \mathrm{C}$ for $3 \mathrm{~min}$ before initiating the reaction by adding mitochondrial protein (5-10 $\mu \mathrm{g}$ protein). The formation of succinyl-CoA was measured by quantifying the increase in absorbance at $235 \mathrm{~nm}$ (Beckman DU-600 spectrophotometer). Controls were run by omitting succinate from the medium.

Immunodetection of succinyl-CoA synthetases in isolate mitochondrial fraction. Mitochondrial extracts were subjected to $(10 \%)$ SDS-PAGE followed by nitrocellulose transfer using a semi-dry Bio-Rad transblot apparatus (25 volts for $45 \mathrm{~min}$ ). Non-specific binding sites were blocked using $5 \%$ non-fat dry milk (either for $1 \mathrm{~h}$ at room temperature or overnight at $4{ }^{\circ} \mathrm{C}$ ). Then the membranes were incubated with either a polyclonal antiserum directed against SCS holoenzyme (1:1000 dilution) or an affinity-purified antisera directed against the common alpha subunit or individual beta subunits of ATP-SCS or GTP- 


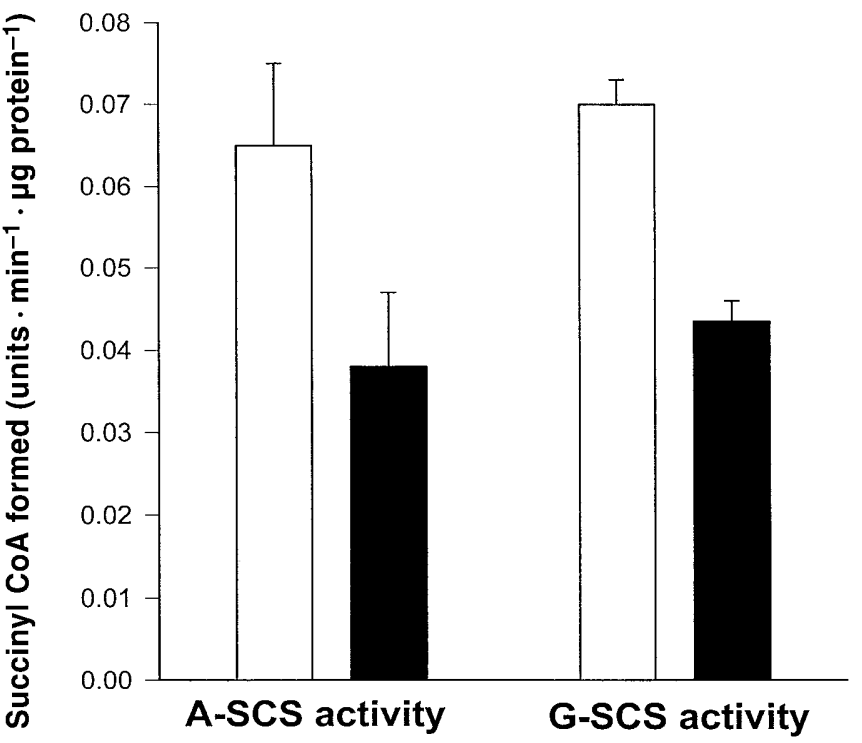

Fig.1. ATP-specific and GTP-specific succinyl-CoA synthetase activities in mitochondrial fractions isolated from HIT-T15 or INS-1 cells: ATP-specific or GTP-specific-succinyl-CoA synthetase activities were measured (see Methods) in mitochondrial fractions from HIT-T15 [ $\square$ ] or INS-1 cells [ם]. Data are means \pm SEM from at least three mitochondrial preparations done in duplicates for each case

SCS (1:200 dilution) for $1 \mathrm{~h}$ at room temperature or for $16 \mathrm{~h}$ at $4{ }^{\circ} \mathrm{C}$ with gentle shaking. Immune complexes were identified using an ECL Plus kit consisting of the anti-rabbit secondary antibody conjugated to peroxidase (1:2000 dilution for $45 \mathrm{~min}$ at room temperature).

Other methods. The protein concentration of samples was assayed by a dye-binding method described previously using bovine serum albumin as a standard [7-12]. The SDS-PAGE was carried out as described previously [7-12].

\section{Results}

The adenine-nucleotide and guanine-nucleotide succinyl-CoA synthetase activities (ATP-SCS and GTPSCS, respectively) were measured spectrophotometrically, in the mitochondrial fractions derived from HIT-T15 and INS-1 cells, as the amount of succinyl$\mathrm{CoA}$ formed in the presence of succinate, CoA, and GTP (or ATP). These enzyme activities were observed in both of the cell types studied (Fig. 1). The ATP-SCS activity was $0.065 \pm 0.01$ and $0.038 \pm 0.009$ units of succinyl-CoA formed $\cdot \mathrm{min}^{-1} \cdot \mu \mathrm{g}$ protein in HIT cell and INS cell mitochondria, respectively. In cognate fractions, the G-SCS activity represented $0.07 \pm 0.003$ in HIT cells and $0.0435 \pm \pm 0.0025$ units in INS cells. These data indicate that the ratio of GTP-specific to ATP-specific activities is near unity in HIT and INS cell mitochondrial fractions. Using a polyclonal antiserum directed against SCS holoenzyme, we identified SCS in HIT cell mitochondrial

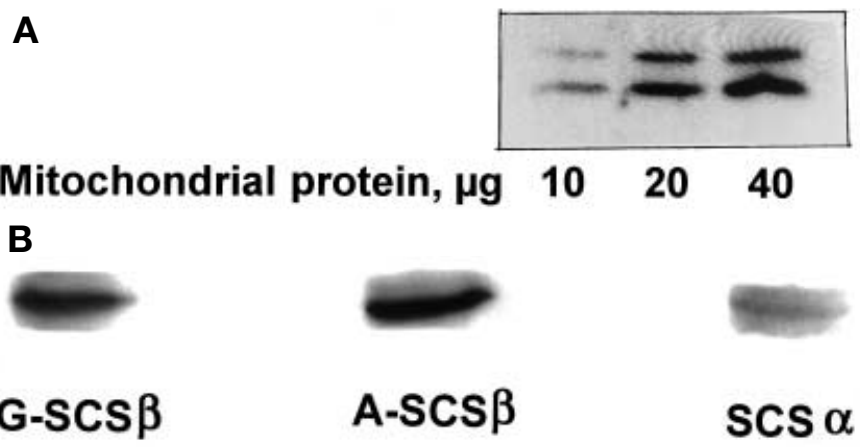

Fig. 2 A-B. Immunonological detection of $\alpha$ and $\beta$ subunits of ATP- and GTP-specific succinyl-CoA synthetases in HIT cell mitochondria: A HIT cell mitochondrial proteins $(10-40 \mu \mathrm{g}$ protein) were separated by SDS-PAGE and transferred onto a nitrocellulose membrane and probed with an antiserum directed against the holoenzyme of porcine heart succinyl CoA synthetase. Similar data were demonstrable in mitochondrial fraction isolated using normal rat islets (data not shown). B HIT cell mitochondrial proteins $(30-40 \mu \mathrm{g}$ protein) were separated by SDS-PAGE and transferred onto a nitrocellulose membrane and probed with affinity purified antisera directed against the alpha and beta subunits of ATP-specific and GTPspecific succinyl-CoA synthetases as indicated in the figure. Data represent at least three individual western blots with identical results

fraction (Fig. 2A). As shown in the figure, this antiserum detected both the $\alpha$ and $\beta$ subunits of SCS. Similar data were found on INS-1 cell as well as normal rat islet mitochondrial fractions (data not shown). It was recently reported that the ATP-sensitive and GTP-sensitive SCS activities share the same $\alpha$ subunit but different beta subunits $[4,5]$. Therefore, we probed the HIT cell mitochondrial fraction for these individual subunits of SCS. Data in Figure 2B demonstrate localization of the alpha and two specific beta subunits in this fraction, confirming the presence of the two isoforms of SCS in the pancreatic beta cell mitochondria. These data are compatible with our data showing the activity of these enzymes in the cognate fractions.

The catalytic function of SCS involves a reversible interchange of GDP (or ADP), succinyl-CoA, and Pi with GTP (or ATP), succinate and CoA through a phosphorylated histidine intermediate [3-5]. Incubation of isolated mitochondrial fraction with $[\gamma-$ $\left.{ }^{32} \mathrm{P}\right]$ ATP resulted in the phosphorylation of a 33000 $\mathrm{M}_{\mathrm{r}}$ protein. Based on the criteria established earlier (e.g., acid and heat lability as well as resistance to alkaline $\mathrm{pH}$ ) $[7,8]$ we tentatively identified the phosphorylated amino acid to be histidine (data not shown). To confirm that the phosphorylated protein represents the alpha subunit of SCS, ${ }^{32} \mathrm{P}$-labelled beta cell proteins were incubated in various concentrations of succinate and coenzyme A $(0-200 \mu \mathrm{mol} / 1$ each). Under these conditions, a concentration-dependent dephosphorylation of the SCS $\alpha$ was ob- 


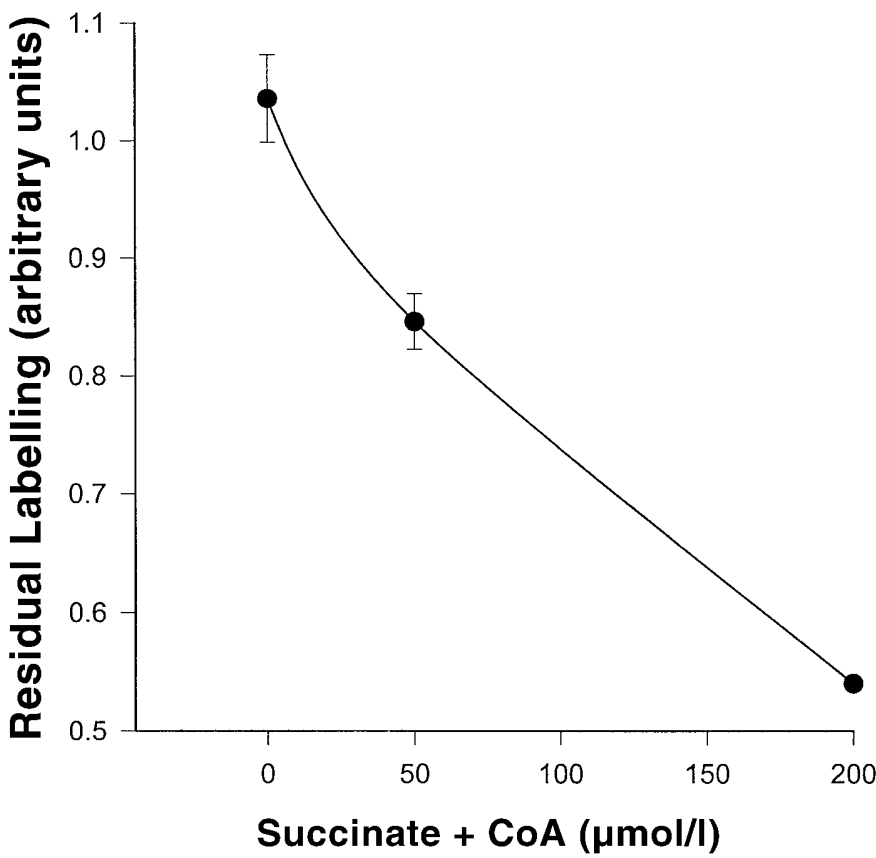

Fig. 3. Dephosphorylation of SCS $\alpha$ subunit in the presence of succinate and CoA: HIT cell mitochondrial proteins were phosphorylated in the presence of $\left[\gamma^{32} \mathrm{P}\right] \mathrm{ATP}$ as indicated in Methods and succinate and CoA were added to the reaction medium at concentrations indicated in the figure. Proteins were separated by SDS-PAGE and degree of residual labelling of SCS alpha subunit was assessed by autoradiography followed by densitometry

served. A near $50 \%$ reduction in the labelling was noticed at $200 \mu \mathrm{mol} / \mathrm{l}$ concentrations of succinate and CoA (Fig.3). Such dephosphorylation, in the presence of exogenous succinate and CoA, was specific only to the $33000 \mathrm{M}_{\mathrm{r}}$ protein, since we observed no effects of these agents on the labelling of other beta cell proteins (data not shown).

\section{Discussion}

Using functional as well as immunological approaches, we localized adenine and guanine nucleotide-specific SCS activities in the mitochondrial fractions from INS and HIT cells. At present there is only one study on the nature of SCS activities in the beta-cell mitochondria [6], which proposed that the insulinotropic properties of monomethyl succinate could result from it increasing the amount of succinyl-CoA and decreasing the concentration of GTP via the SCS reaction. Our study describes the localization of two distinct ATP-specific and GTP-specific SCS in the clonal beta cell. Recently localization of both ATP-specific and GTP-specific SCS activities has been reported in several tissues of pigeons [4,5]. These activities seem to be differentially distributed in various tissues. For example, the ratios of GTP- specific to ATP-specific SCS activities in kidney, brain and heart were 7,1,0.1, respectively. We found a ratio near unity of GTP to ATP-SCS which suggests that the SCS distribution of the beta cell is similar to those observed in the neuronal tissue.

Potentially, SCS could contribute to several functions in the beta-cell mitochondria. It is the only enzyme that generates high-energy phosphate bonds in the form of GTP in the citric acid cycle [14]. Alternatively, GTP is also generated from GDP and ATP by transphosphorylation reactions catalysed by the mitochondrial nucleoside diphosphate kinase (NDPK) which we have characterized in the beta-cell mitochondria $[15,16]$. Therefore, activation of mitochondrial SCS represents a crucial metabolic step for the generation of GTP because, unlike ATP, GTP is not transported across the mitochondrial inner-membrane by the classical nucleotide translocase $[17,18]$. The question is, therefore, what functional roles does GTP have in the mitochondria. GTP is required to activate specific mitochondrial G-proteins which have been implicated in several mitochondrial functions including, protein synthesis, steroidogenesis, protein transport, membrane fusion, contact point alignment and permeability transition [19-24]. Whereas several studies, including our own, implied G-proteins in physiological insulin secretion [25-29], no detailed studies have been carried out so far on the localization of G-proteins in the beta-cell mitochondria. In this context, two recent studies from our laboratory provided (albeit indirect) evidence supporting the existence of G-proteins in the mitochondria-enriched fractions isolated from normal rat islets and clonal beta cells. In the first, we have found considerable amounts of high-affinity GTPase activity in normal rat islet mitochondrial fraction [11]. In a second study, we observed the enrichment of prenylcysteine methyltransferase activity in the mitochondria-rich fraction derived from INS-1 cells; this enzyme carboxyl methylates and activates specific Gproteins in a GTP-sensitive manner [10].

Recently, using isolated beta cells, we identified two proteins that could contribute to the activation of islet endogenous G-proteins. They are the NDPK and the $\beta$ subunit of trimeric G-proteins [7-9]. We have shown that both of these proteins underwent phosphorylation at a histidine residue and that phosphate, in turn, is transferred to either free GDP or GDP bound to the G-proteins (inactive conformation), yielding free GTP or GTP-bound to G-proteins (active conformation). In the current studies, based on the stability characteristics (acid-lability and heat-lability and base-stability), we have identified, to some extent, that the phosphorylated amino acid of SCS is a histidine. This is compatible with data showing that SCS undergoes phosphorylation at a histidine residue [3]. We have no data to suggest a phosphotransfer from P-His of SCS to various accep- 
tors; however, recent studies [30] have indicated that SCS from Trypanosoma brucei undergoes transient phosphorylation at a histidine residue and that phosphate is rapidly transferred to appropriate acceptors. It remains to be established whether the mitochondrial SCS could calatyse such phosphotransfer from phosphohistidine similar to the mechanisms we described for NDPK and beta subunit of trimeric GTPases in the islet beta cell [7-9].

It was thought that as an intermediate step of its catalytic cycle, the alpha subunit of SCS undergoes autophosphorylation at a histidine residue. Recent evidence indicates, however, that such a phosphorylation step could be mediated or potentiated or both by a histidine kinase-like enzyme, such as NDPK $[31,32]$. For example, it was suggested that SCS stays as a complex with NDPK and trace amounts of NDPK seem to stimulate the activity of SCS [31]. Using sucrose-density gradient centrifugation methods, other investigators have also shown the association of NDPK with SCS in isolated mitochondrial fraction [33]. Compatible with these observations, using isolated beta-cell mitochondrial fractions, we recently reported the association of NDPK with SCS [16]. Together, our current data provide the basis for future studies on the specific interaction (and possible cross-talk) among various mitochondrial proteins (e.g., mNDPK and SCS) and to show the roles of these proteins (and protein histidine phosphorylation) in the stimulus-secretion coupling of the pancreatic beta cell leading to insulin secretion.

We provide evidence for the localization of two distinct (ATP-specific and GTP-specific) succinylCoA synthetases in the islet beta-cell mitochondria. It is well established that ATP is critical for the betacell mitochondrial metabolism and we propose that GTP generated by the activation of SCS could contribute to key functional roles in the mitochondrial metabolism, including activation of mitichondrial GTP-binding proteins, leading to insulin secretion. Alternatively, like ATP, the intramitochondrial GTP could provide signal functions independent from interaction with and activation of the beta-cell G-proteins, to facilitate insulin secretion [34]. Such potential intracellular functions and roles of GTP, in the context of insulin secretion, have recently been reviewed [35].

Acknowledgements. This research is supported by funds from the Department of Veteran Affairs and the American Diabetes Association. I wish to thank H.-Q. Chen, L. Modrick and Y. Lyles-Grandison for their excellent technical help and Dr. Robertson and Dr. Wollheim for providing HIT-T15 and INS1 cells, respectively. I very much appreciate the help and support of Dr. Lambeth for generously providing us with affinitypurified antisera directed against the alpha subunit of SCS and beta subunits of ATP-specific and GTP-specific SCS and valuable discussions and suggestions throughout the course of these studies. A generous gift of anti-SCS serum by Dr. Bridger and Dr. Woldoko is acknowledged. Part of this work was presented at the Third International Congress on Genetics, Biochemistry and Physiology of Nucleoside Diphosphate Kinase in Bordeaux, France (Sept. 1999), and at the 60 th Annual Meetings of the American Diabetes Association in San Antonio, Texas, USA (June, 2000).

\section{References}

1. Ottaway JH, McClellan JA, Saunderson CL (1981) Succinic thiokinase and metabolic control. Int J Biochem 13: 401-410

2. Labbe RF, Kurumada T, Onisawa J (1965) The role of succinyl-CoA synthetase in the control of heme biosynthesis. Biochim Biophys Acta 111: 403-415

3. Bridger WA, Wolodko WT, Henning W, Upton C, Majumdar R, and Williams SP (1987) The subunits of succinyl-coenzyme A synthetase-function and assembly. Biochem Soc Symp 54: 103-111

4. Johnson, JD, Muhonen WW, Lambeth, DO (1998) Characterization of the ATP-and GTP-specific succinyl-CoA synthetases in pigeon. The enzymes incorporate the same subunit. J Biol Chem 273: 27573-27579

5. Johnson, JD, Mehus, JG, Tews K, Milavetz BI, Lambeth DO (1998) Genetic evidence for the expression of ATPand GTP-specific succinyl-CoA synthetases in multicellular eucaryotes J Biol Chem 273: 27580-27586

6. Fahien LA, MacDonald MJ, Kmiotek EH, Mertz RJ, Fahien CM (1988) Regulation of insulin release by factors that also modify glutamate dehydrogenase. J Biol Chem 263: $13610-13614$

7. Kowluru A, Seavey SE, Rhodes CJ, Metz SA (1996) A novel regulatory mechanism for trimeric GTP-binding proteins in the membrane and secretory granule fractions of human and rodent cells. Biochem J 313: 97-107

8. Kowluru A, Metz SA (1994) Characterization of nucleoside diphosphokinase activity in human and rodent pancreatic cells: evidence for its role in the formation of guanosine triphosphate, a permissive factor in nutrient-induced insulin secretion. Biochemistry 33: 12495-12503

9. Kowluru A, Seavey SE, Rabaglia ME , Metz SA (1995) Non-specific stimulatory effects of mastoparan on pancreatic islet nucleotide diphosphokinase activity: Dissociation from insulin secretion. Biochem Pharmacol 49: 263-266

10. Li G, Kowluru A, Metz SA (1996) Characterization of prenylcysteine methyltransferase in insulin-secreting cells. Biochem J 316: 345-351

11. Kowluru A, Metz SA (1994) Stimulation by prostaglandin $\mathrm{E}_{2}$ of a high-affinity GTPase in the secretory granules of normal rat and human pancreatic islets. Biochem J 297: 399- 406

12. Kowluru A, Rabaglia, ME, Muse KE, Metz SA (1994) Subcellular localization and kinetic characterization of guanine nucleotide binding proteins in normal rat and human pancreatic islets and transformed cells. Biochim Biophys Acta 1222: 348-359

13. Cha S, Parks RE Jr (1964) Succinic thiokinase. Purification of the enzyme from pig heart. J Biol Chem 239: 1961-1967

14. Stryer L (1999) In: Biochemistry, WW. Freeman and Company, New York, pp 511

15. Kowluru A (1999) High-energy phosphate metabolism in the pancreatic beta cell: studies of mitochondrial nucleoside diphosphokinase and succinyl CoA synthetase. In Proceedings of the Third International Congress of the Genetics, Biochemistry and Physiology of nm23/nucleoside diphosphate kinase Sept. 1999; Bordeaux, France. T12 p 
16. Kowluru A, Milon L, Lacombe ML (2000) Identification of $\mathrm{nm} 23-\mathrm{H} 4$, the mitochondrial isoform of nucleoside diphosphate kinase NDPK. in the pancreatic cell. Diabetes 49: [Suppl 1]: A 250, (Abstract)

17. Klingenberg M, Pfaff E (1968) Metabolic control in mitochondria by adenine nucleotide translocation. Biochem Soc Symp 27: 105-122

18. Pfaff E, Klingenberg M, Heldt HW (1965) Unspecific permeation and specific exchange of nucleotides in liver mitochondria. Biochim Biophys Acta 104: 312-314

19. Thomson M (1998) What are guanosine triphosphate-binding proteins doing in mitochondria. Biochim Biophys Acta 1403: 211-218

20. Thomson M, Lim G, Hall PF, Kuyznierewicz I(1998) Overlay blot identification of GTP- binding proteins in mitochondria from human placenta. Placenta 19: 209-215

21. Hegde R (1998) The 24-kDa subunit of the bovine mitochondrial NADH: ubiquinone oxidoreducatse is a G-protein. Biochem Biophys Res Commun 244: 620-629

22. Denslow ND, Anders JC, O'Brien TW (1991) Bovine mitochondrial ribosomes possess a high- affinity binding site fr guanine nucleotides. J Biol Chem 266: 9586-9590

23. Susin SA, Zamzani N, Kroemer G (1998) Mitochondria as regulators of apoptosis: Doubt no more. Biochim Biophys Acta 1336: 151-165

24. Crompton M (1999) The mitochondrial permeability transition pore and its role in cell death. Biochem $\mathbf{J} 341$ : 233-249

25. Kowluru A, Seavey SE, Li G et al. (1996) Glucose- and GTP-dependent stimulation of the carboxylmethylation of $\mathrm{Cdc} 42$ in rodent and human pancreatic islets and pure cells. J Clin Invest 98: 540-555

26. Kowluru A, Li G, Metz SA (1997) Glucose activates the carboxyl methylation of subunits of trimeric GTP-binding proteins in pancreatic cells. J Clin Invest 100: 1596- 1610
27. Kowluru A, Rabaglia ME, Muse KE, Metz SA (1994) Subcellular localization and kinetic characterization of guanine nucleotide binding proteins in normal rat and human pancreatic islets and transformed cells. Biochim Biophys Acta 1222: 348-359

28. Sharp GW (1996) Mechanism of inhibition of insulin release. Am J Physiol 271: C1781-C1799

29. Robertson RP, Seaquist ER, Walseth TF (1991) G-proteins and modulation of insulin secretion. Diabetes 40: 1-6

30. Hunger-Glaser I, Linder M, Seebeck T (1999) Inhibition of succinyl -CoA synthetase histidine-phosphorylation in Trypanosoma brucei by an inhibitor of bacterial two-component systems. Mol Biochem Parasitol 100: 43-52

31. Kavannaugh-Black A, Connolly DM, Chugani SA, Chakrabarty AM (1994) Characterization of nucleoside diphosphate kinase from $P$. Aeruginosa: Complex formation with succinyl-CoA synthetase. Proc Natl Acad Sci USA 91: 5883-5887

32. Wagner PD, Steeg PS, Vu N-D (1997) Two component kinase-like activity of nm23 correlates with its motility suppressing activity. Proc Natl Acad Sci (USA) 94: 9000-9005

33. Kadramas EF, Ray, P.D., Lambeth, DO (1991) Apparent ATP-linked succinate thiokinase activity and its relation to nucleoside diphosphate kinase in mitochondrial matrix preparations from rabbit. Biochim Biophys Acta 1074: 339-346

34. Zunkler BJ, Lenzen S, Penten U (1986) Glucose enhances GTP content in mouse pancreatic islets. IRCS Medical Sciences 14: 354-355

35. Kowluru A, Metz SA (1994) GTP and its binding proteins in the regulation of insulin exocytosis. In: Draznin B, LeRoith D (eds) Molecular Biology of Diabetes. Humana Press, Totowa, pp 249-283 